\title{
Japanese Randomized Trial for Investigation of a Combined Therapy of Amiodarone and Implantable Cardioverter Defibrillator in Patients With Ventricular Tachycardia and Fibrillation The Nippon ICD Plus Pharmacologic Option Necessity (NIPPON) Study Design
}

\author{
Takashi Kurita ${ }^{1}$; Hideo Mitamura ${ }^{2}$; Yoshifusa Aizawa ${ }^{3}$; Takashi Nitta ${ }^{4}$ Kazutaka Aonuma ${ }^{5}$; \\ Naoya Tsuboi ${ }^{6}$; Masaomi Chinushi ${ }^{7}$; Yoshinori Kobayashi ${ }^{8}$; Kyoko Soejima ${ }^{9}$; \\ Kazuhiro Satomi ${ }^{1}$; Hiroshi Furushima ${ }^{3}$; Tohru Ohe ${ }^{10}$; Satoshi Ogawa ${ }^{9}$; \\ Itsuo Kodama ${ }^{11}$; Hiroshi Ohtsu ${ }^{12}$; Tsutomu Yamazaki ${ }^{12}$ \\ (Nippon ICD Plus Pharmachologic Option Necessity (NIPPON) Investigators)
}

\begin{abstract}
Background Implantable cardioverter-defibrillators (ICDs) are the most effective therapy in reducing the mortality of patients with life-threatening ventricular tachyarrhythmias. However, the ICD cannot prevent the recurrence of tachycarida attacks and that limits the clinical usefulness of them. The Nippon ICD Plus Pharmachologic Option Necessity (NIPPON) trial was designed as the first prospective randomized study to test the hypothesis whether amiodarone could improve the patient's clinical outcome by reducing the amount of ICD therapy in the Japanese patient population.

Methods and Results Approximately 400 patients with organic heart disease and spontaneous episode(s) of sustained ventricular tachycardia/fibrillation (VT/VF) will be randomly assigned to one of 2 groups; the amiodarone group and non-amiodarone group. Both groups of patients will be followed at least for 24 months. The end-point committee will adjudicate events in a blinded fashion. The primary end-points of this study are determination of the appropriate therapy from the ICD and alteration of the assigned treatment because of its harmful effects and/or frequent ICD therapies.

Conclusion The NIPPON study is expected to confirm our understanding of the prognostic and therapeutic usefulness of adjuvant amiodarone therapy for patients with an ICD and with a history of sustained VT/VF. (Circ J 2006; 70: 316-320)
\end{abstract}

Key Words: Amiodarone; Implantable cardioverter defibrillator; Ventricular fibrillation; Ventricular tachycardia

I mplantable cardioverter-defibrillators (ICDs) are the most effective therapy in reducing the mortality of patients who have a history of life-threatening ventricular tachyarrhythmias when compared with the effects

(Received September 28, 2005; revised manuscript received December 12, 2005; accepted January 11, 2006)

${ }^{1}$ Department of Cardiovascular Medicine, National Cardiovascular Center, Suita, ${ }^{2}$ Department of Cardiology, Saiseikai Central Hospital, Tokyo, ${ }^{3}$ First Department of Internal Medicine, Niigata University School of Medicine, Niigata, ${ }^{4}$ Department of Biological Regulation and Regenerative Surgery, Nippon Medical School, Graduate School of Medicine, Tokyo, ${ }^{5}$ Department of Internal Medicine, Cardiovascular Division, University of Tsukuba, Tsukuba, ${ }^{6}$ Department of Cardiology, Social Insurance Chukyo Hospital, Nagoya, ${ }^{7}$ School of Health Science, Niigata University School of Medicine, Niigata, ${ }^{8}$ First Department of Internal Medicine, Nippon Medical School, Tokyo, ${ }^{9}$ Cardiopulmonary Division, Keio University School of Medicine, Tokyo, ${ }^{10}$ Department of Cardiovascular Medicine, Okayama University, Okayama, ${ }^{11}$ Department of Circulation, Research Institute of Environment Medicine, Nagoya University, Nagoya, ${ }^{12}$ Department of Clinical Bioinformatics, University of Tokyo, Tokyo, Japan

Mailing address: Takashi Kurita, MD, Department of Cardiovascular Medicine, National Cardiovascular Center, 5-7-1 Fujishirodai, Suita 565-8565, Japan. E-mail: kuritat@hsp.ncvc.go.jp of antiarrhythmic drugs $!^{-7}$ However, the ICD cannot prevent the recurrence of ventricular tachycardia/fibrillation (VT/VF) attacks and potential loss of consciousness and that limits the clinical usefulness of it.

An important rationale for an adjuvant therapy with antiarrhythmic drugs in patients with ICDs is that defibrillators do not provide absolute protection against death from arrhythmias. Approximately 2 percent of VT/VF episodes were reported to be refractory to appropriate ICD therapy; the condition is known as 'electrical storm' or 'pulse-less electrical activity'? Recent studies suggest that episodes of $\mathrm{VF}$, even ones that are successfully terminated by an ICD, may increase the risk of myocardial and cerebral ischemic injury9-11 The avoidance of frequent shocks by the use of antiarrhythmic agents may be crucial for the safety and quality of life (QOL) of patients with ICDs. Another aim for the use of a combination drug therapy with the ICD is to reduce shock therapies not only by inhibiting ventricular tachyarrhythmias, per se, but also by converting rapid $\mathrm{VT} / \mathrm{VF}$ to hemodynamically stable VT, which may not only be more responsive to anti-tachycardia pacing but can also avoid loss of consciousness of the patient!2 Further- 
NIPPON Study Design

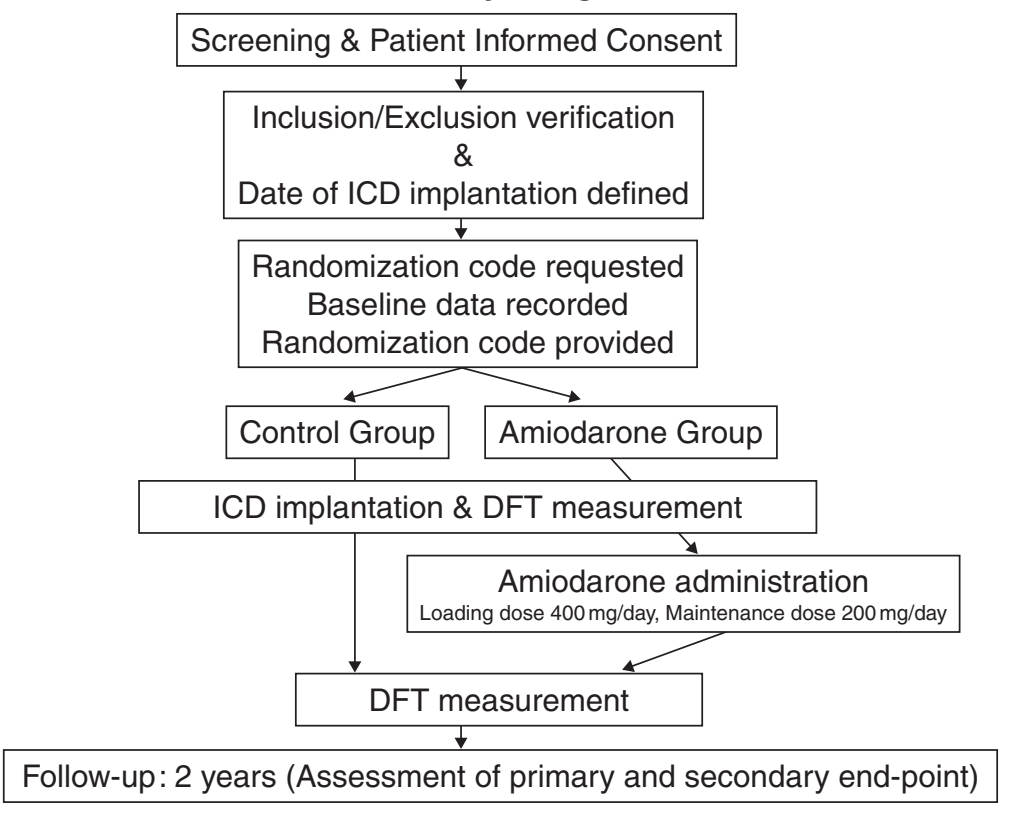

Fig 1. Diagram of the Nippon ICD Plus Pharmacologic Option Necessity (NIPPON) study design. Randomization would be performed before ICD implantation. ICD, implantable cardioverter-defibrillator; DFT, defibrillation threshold. more, an inappropriate ICD therapy may also be prevented by antiarrhythmic drugs through the suppression of supraventricular tachyarrhythmias.

In contrast, the combined use of antiarrhythmic agents with ICDs might lead to adverse responses such as an unacceptable increase in the defibrillation threshold (DFT) and under-detection of VT/VF because of a prolongation of an arrhythmia cycle length beyond the programmed detection interval, and potential proarrhythmias or extracardiac toxicity ${ }^{13-15}$ The Nippon ICD Plus Pharmachologic Option Necessity (NIPPON) pre-study demonstrated that the VT/VF event rate in ICD patients with class I agent adjunctive therapy was significantly higher than that in patients with amiodarone! 6 Furthermore, no fatal side-effect of amiodarone was observed in the NIPPON pre-study.

Sotalol has been proven to be safe and efficacious in reducing the risk of death and frequency of defibrillator shocks. ${ }^{17-19}$ Amiodarone is the most frequently prescribed drug for ICD patients in Japan, but its efficacy to suppress VT/VF episodes in ICD patients has not yet been substantiated ${ }^{16,19}$ The Cardiac Arrest in Seattle: Conventional Versus Amiodarone Drug Evaluation (CASCADE) trial demonstrated that amiodarone prevents potentially lethal arrhythmias in patients resuscitated from cardiac arrest compared with conventional antiarrhythmic drug therapy guided by electrophysiological study or Holter monitoring? ${ }^{20}$ However, that study was not designed to confirm the efficacy of anti-arrhythmic drugs in reducing VT/VF recurrences in patients with a history of VT/VF and with ICD therapy.

The NIPPON trial was designed to be the first prospective randomized study to test the hypothesis that amiodarone could improve the patient's clinical outcome by reducing ICD therapy deliveries required for prompt termination of VT/VF in the Japanese patient population.

\section{Methods}

\section{Patient Population}

The patients entering this trial will meet the following entry criteria: (1) all patients will have spontaneous epi- sode(s) of sustained VT or VF; (2) all patients will have organic heart disease as documented either by electrocardiography, echocardiography, cardiac catherterization, nuclear scintigraphy, computed tomography or magnetic resonance imaging; (3) all patients will correspond to the guidelines for ICD therapy determined by the Ministry of Health, Labor and Welfare, in Japan; (4) all patients will be free of any class I or class III drugs at the time of the ICD implantation, and all anti-arrhythmic drugs (except for $\beta$ blockers) will be discontinued at least 5 half-lives before the implantation. Beta-blockers will be allowed to be prescribed when appropriate.

The exclusion criteria for this study are when patients have the following: (1) idiopathic VT/VF; (2) Brugada syndrome; (3) congenital long QT syndrome; (4) an incessant form of VT/VF; (5) VT/VF resulting from a transient or reversible disorder such as acute myocardial infarction, electrolyte imbalance or drugs; (5) are aged less than 20 years old; (6) are taking class I or class III drugs (including amiodarone), which cannot be discontinued; (7) a life expectancy less than 2 years; (8) congestive heart failure with a New York Heart Association (NYHA) cardiac function class IV or are candidates for cardiac transplantation; (9) bradycardia less than 50 beats/min.; (10) second- or third-degree atrioventricular block; (11) corrected QT interval $(\mathrm{QTc}=\mathrm{QT} \sqrt{\mathrm{RR}} \mathrm{s})$ equal to or longer than $480 \mathrm{~ms}$; (12) significant pulmonary disease such as interstitial pneumonitis, pulmonary fibrosis, diffusion capacity of the lung for carbon monoxide (DLco) $<60 \%$ or high molecular weight mucin-like antigen (KL-6) >1,000 U/ml; (13) severe dysfunction of the liver, kidney or thyroid; (14) more than 2 episodes of hemodynamically unstable sustained VT or VF per month before the ICD implantation; (15) are candidates for curative procedures for VT/VF such as catheter ablation or cryosurgery; (16) unstable angina pectoris; (17) DFT at the ICD implantation of more than $20 \mathrm{~J}$; and (18) are pregnant (or have the possibility of), or are breast feeding. 
Defibrillation test protocol during implantation

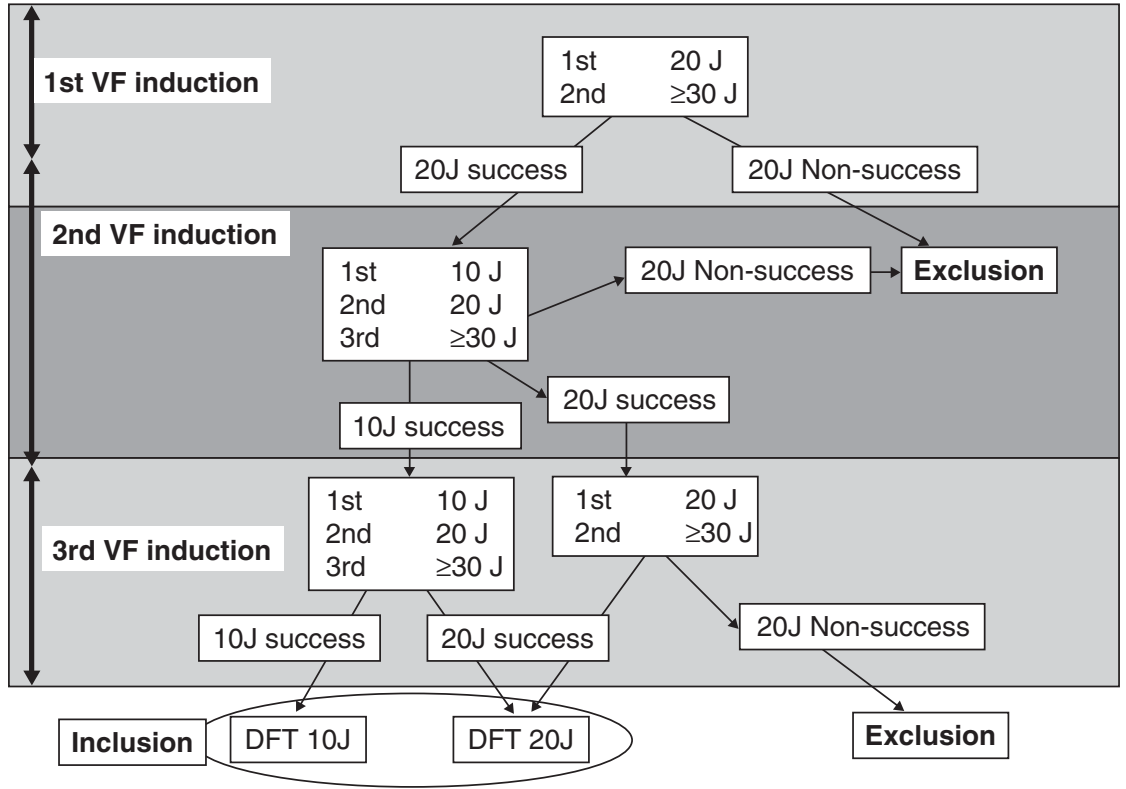

Defibrillation test protocol after implantation

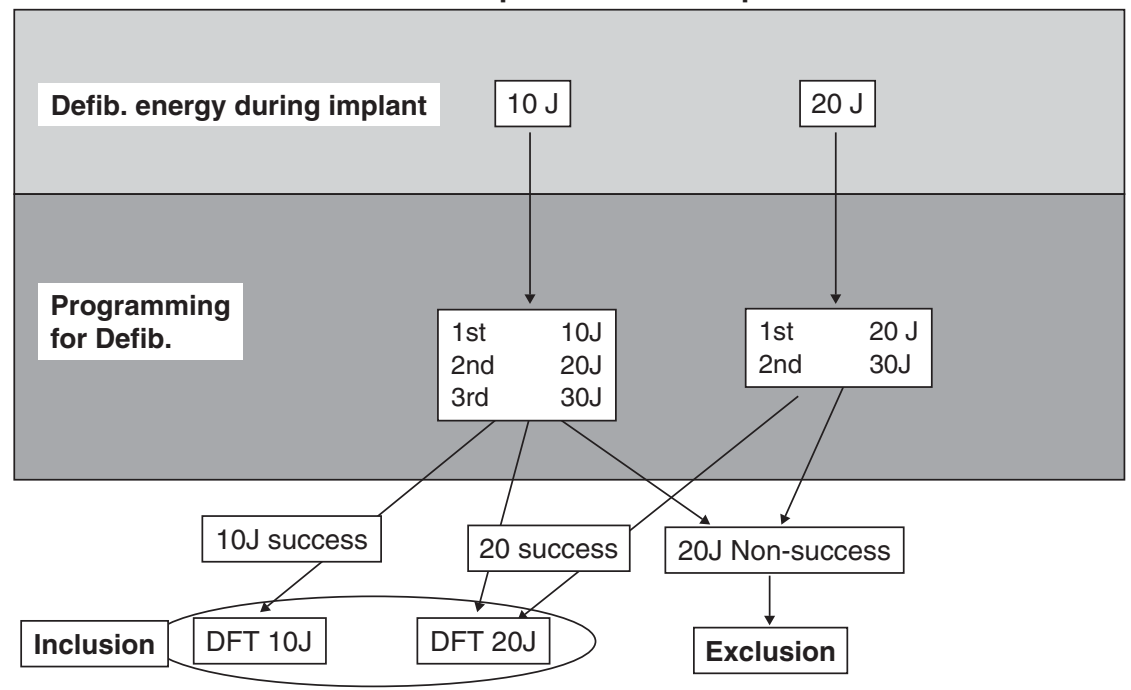

Fig 2. Diagram of the defibrillation test during ICD implantation. An almost exact defribrillation threshold (DFT) will be determined by inducing VF 3 times. ICD, implantable cardioverter-defibrillator; Success, successful defibrillation; Non-success, unsuccessful defibrillation; VF, ventricular fibrillation. This diagram is designed for a device with a maximum shock output of 30 J.

Fig 3. Diagram of the defibrillation threshold (DFT) measurements after ICD implantation. A series of deliverable shock energies will be programmed individually according to the DFT during ICD implantation. A decrease in the DFT will not be assessed by using this protocol. ICD, implantable cardioverter defibrillator; Success, successful defibrillation; Non-success, unsuccessful defibrillation; VF, ventricular fibrillation; Defib, defibrillation. This diagram is designed for a device with a maximum shock output of $30 \mathrm{~J}$.

\section{End-Points}

Primary End-Points The NIPPON study will investigate the efficacy of amiodarone in reducing the appropriate ICD therapy deliveries and in improving the QOL in patients with spontaneous VT/VF episodes caused by organic heart disease. The primary end-points of this study are: (1) an appropriate ICD therapy; and (2) an alteration of the assigned treatment because of its harmful effect and/or frequent ICD therapy deliveries (more than 3 sessions of ICD first therapy within $24 \mathrm{~h}$ ). The latter one includes termination of amiodarone or a transition to an alternative antiarrhythmic drug (class I or class III) in the amiodarone group, addition of class I or class III drugs in the control group, or curative interventions such as catheter ablation or cryosurgery in either group.

\section{Secondary End-Points}

The secondary end-points of this study are: (1) total death; (2) arrhythmic death; (3) cardiac death; (4) re-hospitalization aiming to control VT/VF; (5) impairment of patient's QOL; (6) deterioration of heart failure (worsening of NYHA class or unplanned admission to the hospital because of heart failure); (7) abnormal increase in the DFT (less than a $10 \mathrm{~J}$ safety margin); (8) inappropriate ICD therapy; or (9) occurrence of side-effects from amiodarone.

\section{Study Design}

The NIPPON study is a multicenter randomized, notreatment concurrent controlled study to access the efficacy of amiodarone in patients with an ICD.

After obtaining written informed consent, patients will be randomly assigned to one of 2 groups; amiodarone group and non-amiodarone group. An ICD (basically, dual chamber types) will be implanted as soon as possible after randomization (Fig 1). At least a 10-J safety margin for defibrillation will be determined by inducing VF 3 times 
under the protocol (Fig 2). As a general rule, amiodarone will be started on the next day after the ICD implantation with a loading dose of $400 \mathrm{mg} /$ day. Two weeks after the loading dose period, amiodarone will be reduced to a maintenance dose of $200 \mathrm{mg} /$ day. The defibrillation ability will be reassessed 2-4 weeks after the ICD implantation in both groups (Fig 3). The aim of the second test is to recognize at least a 10-J safety margin for defibrillation, and therefore, an improvement of the DFT will not be evaluated. Any patients, in whom the DFT has increased to a level where the safety margin is less than $10 \mathrm{~J}$ during the second test, will be excluded. All patients will be followed up for at least 2 years in cases where the patient is still alive.

Discontinuation of amiodarone will be considered if the following conditions are observed: (1) a marked QT interval prolongation (QTc interval $>550 \mathrm{~ms}$ ); (2) symptoms suggestive of pulmonary intoxication; (3) newly developed infiltration on the chest X-ray; (4) a DLco of less than $60 \%$ for the absolute value or a $20 \%$ decrease in the control value; or (5) other adverse effects from amiodarone such as frequent VT/VF, congestive heart failure.

The patient's QOL will be evaluated by a questionnaire comprised of general health-related questions and also ICD-specific questions. The general health-related questions are based on the SF-36 ${ }^{21}$ Furthermore, several additional questions concerned with arrhythmias and the ICD implantation have been specially designed by the NIPPON committee, and those consist of only 4 questions asking about the patient's anxiety and restrictions on daily living related to the VT/VF itself and the assigned treatment of ICD implantation with or without amiodarone.

\section{Patient Follow-up}

All patients will be followed up at the ICD clinic in each participating institution every 3 months, and the therapy recordings retrieved from the ICD will be analyzed in terms of heart rate, heart rate variability, arrhythmia morphology and temporal relation between the atrial and ventricle electrograms. The side- effects of amiodarone will be assessed by symptoms, chest X-ray, DLco, and KL-6 level every 3 months, ${ }^{2}$ and the QOL will be evaluated before randomization and at 12 and 24 months after the initiation of the treatment.

\section{Recommendations for the ICD System and Optimal Programming}

Although the selection of the ICD system will be left to the judgment of the physician, a dual chamber ICD approved by the Ministry of Health, Labor and Welfare, of Japan, at the time of the implantation will be recommended, because the dual chamber device provides much more exact information for the discrimination of the tachycardia and an appropriate back-up pace.

The device will be programmed to maximize its ability for the detection and termination of the tachyarrhythmias. The detection interval for VT will be programmed according to the slowest VT with a safety margin of more than $60 \mathrm{~ms}$ combined with an algorithm for the discriminative criteria of atrio-ventricular dissociation, cycle length variation and acceleration of the tachycardia.23,24 The detection interval for VF will be programmed equal to or more than $300 \mathrm{~ms}$, and the maximum shock energy will be selected for all defibrillation therapies.

The DDI mode for backup pacing will be programmed to provide a relatively long $\mathrm{A}-\mathrm{V}$ delay, hence allowing the atrioventricular propagation of the atrial impulse to avoid asynchronous contractions because of artificial right ventricular pacing. 25

\section{Data Analysis and Sample Size}

The primary analysis will be made on an unadjusted intention-to-treat comparison between groups for the time to any part of the composite primary end-point (appropriate therapy from the ICD or alteration of the assigned treatment) and secondary end-point (total death, arrhythmic death, cardiac death or re-hospitalization aiming to control VT/VF) by using the Kaplan-Meier survival curve and log-rank test. As part of the secondary end-point, a questionnaire for the assessment of the patient's QOL will be collected from each group, and the absolute values for each question will be used to calculate the mean value, SD, number of cases, and median value (as required) per question per group. The inter-group difference will be assayed at each measurement point by using the unpaired Student's t-test or Mann-Whitney U-test. Differences between the groups and over time in the absolute values of the measurement points will be investigated by repeated ANOVA. Items that cannot be appropriately assessed and analyzed by absolute values will be subjected to separate analysis by the most appropriate method. The patient background factors and other observational items will be aggregated by group, and any inter-group differences will be analyzed by methods corresponding to the nature of the data.

Two hundred patients in each group (a total 400 patients) will be enrolled at more than 40 Japanese centers over a 3-year period with the minimum follow-up of 2 years. Therefore, we plan for this study to last a total of 5 years. The target number of cases has been established by our estimation of the primary end-point incidence26 The projected incidence of appropriate ICD therapy in the nonamiodarone group is estimated to be $40 \%$ over 3.5 years, and the expected event decrease rate in the amiodarone group is estimated to be $30 \%$; these were presumed based on the CASCADE and NIPPON pre-study! 16,20 By using these outcome rates, and assuming an $\alpha$ error of 0.05 , we will have a power of above 0.80 for demonstrating this outcome if a total of 400 patients are randomized. Assuming further that approximately $10 \%$ of patients will be found ineligible after randomization, the total sample size needed in this trial is 440 patients.

\section{Steering Committee}

This committee will be responsible for developing and monitoring the implementation of the protocol. The committee is supported by a statistician, independent of the sponsor, and is responsible for ensuring the timely publication of the results.

\section{Adverse Event and End-Point Committee}

This committee will be responsible for classifying all deaths that could potentially contribute to an end-point or adverse event. When accuracy of the ICD therapy during the tachycardia is inconclusive, retrieved electrograms from the device will be analyzed by the committee. All these events will be adjudicated in a blinded manner.

\section{Data Safety Monitoring Board (DSMB)}

The DSMB will be responsible for monitoring patient safety and will recommend that the trial be stopped prematurely if a pre-defined increase in all-cause mortality occurs 
in the amiodarone arm or if a 3-standard deviation in the benefit in all-cause mortality, on a log rank test, is observed.

\section{Randomization}

Randomization of patients will be through a centralized, concealed, minimization process implemented by an independent statistician.

\section{Expected Implications}

The NIPPON study is expected to confirm our understanding of the prognostic and therapeutic usefulness of adjuvant amiodarone therapy for patients with an ICD, and with a history of sustained VT/VF. There are 4 supplemental features in this trial to be addressed. First, this trial will allow us to use amiodarone based on a more scientific background than an individual's anecdotal experience, thus should help our clinical strategy aiming to reduce the VT/VF recurrence and improve the mortality and/or QOL. Second, the efficacy and feasibility, as well as safety, of a relatively low dose of amiodarone, which has been traditionally preferred in Japan, will be properly assessed. Third, this trial will enable the evaluatation of the role of KL-6 in the early detection of pulmonary intoxication. Finally, the study will generate the first large database of Japanese ICD patients, which can be used for subgroup analysis, and provide further information for investigating the peculiar characteristics of VT/VF patients in Japan.

\section{Acknowledgement}

This study was partly supported by the Japan Heart Foundation. The NIPPON study is a project planned by The Japanese Society of Electrocardiology. All designs and protocols of the NIPPON trial have been accepted by the Executive Board of The Japanese Society of Electrocardiology.

\section{References}

1. Domanski MJ, Sakseena S, Epstein AE, Hallstrom AP, Brodsky MA, Kim S, et al. Relative effectiveness of the implantable cardioverterdefibrillator and antiarrhythmic drugs in patients with varying degrees of left ventricular dysfunction who have survived malignant ventricular arrhythmias: AVID Investigators: Antiarrhythmics Versus Implantable Defibrillators. J Am Coll Cardial 1999; 34: 1090-1095.

2. Sheldon R, Connolly S, Krahn A, Roberts R, Gent M, Gardner M. Identification of patients most likely to benefit from implantable cardioverter-defibrillator therapy: The Canadian Implantable Defibrillator Study. Circulation 2000; 101: 1660-1664.

3. Connolly SJ, Gent M, Roberts RS, Dorian P, Roy D, Sheldon RS, et al. Canadian implantable defibrillator study (CIDS): A randomized trial of the implantable cardioverter defibrillator against amiodarone. Circulation 2000; 101: 1297-1302.

4. Kuck KH, Cappato R, Siebels J, Ruppel R. Randomized comparison of antiarrhythmic drug therapy with implantable defibrillators in patients resuscitated from cardiac arrest: The Cardiac Arrest Study Hamburg (CASH). Circulation 2000; 102: 748-754.

5. Connolly SJ, Hallstrom AP, Cappato R, Schron EB, Kuck KH, Zipes DP, et al. Meta-analysis of the implantable cardioverter defibrillator secondary prevention trials. Eur Heart J 2000; 21: 2071-2078.

6. Desai AS, Fanf JC, Maisel WH, Baughman KL. Implantable defibrillators for the prevention of mortality in patients with nonischemic cardiomyopathy. JAMA 2004; 292: 2874-2879.

7. Richter S, Duray G, Gronefeld G, Israel CW, Hohnloser SH. Preven- tion of sudden cardiac death: Lessons from recent controlled trials. Circ J 2005; 69: 19-22.

8. Pacifico A, Johnson J, Stanton M. Comparison of results in two implantable defibrillators. Am J Cardiol 1998; 82: 875-880.

9. Tokano T, Bach D, Chang J. Effect of ventricular shock strength on cardiac hemodynamics. J Cardiovasc Electrophysiol 1998; 9: 791 797.

10. Joglar J, Kessler D, Welch P. Effects of repeated electrical defibrillations on cardiac troponin I levels. Am Heart J 1999; 83: 270-272.

11. de Vries J, Bakker P, Visser G, Diephuis J, van Huffelen A. Changes in cerebral oxygen uptake and cerebral electrical activity during defibrillation threshold testing. Anesth Analg 1998; 87: 16-20.

12. Ohhira K, Niwano S, Furushima H, Taneda K, Chinushi M, Aizawa $\mathrm{Y}$. The use of the block cycle length as a safe and efficient means of interrupting sustained ventricular tachycardia and its pharmacological modification. Pacing Clin Electrophysiol 1998; 21: 1686-1692.

13. Kuhlkamp V, Mewis C, Suchalla R, Merami J, Domberger V, Seipel L. Effect of amiodarone and sotalol on the defibrillation threshold in comparison to patients without antiarrhythmic drug treatment. Int $J$ Cardiol 1999; 69: 271-279.

14. Pelosi F Jr, Oral H, Kim MH, Sticherling C, Horwood L, Knight BP, et al. Effect of chronic amiodarone therapy on defibrillation energy requirements in humans. J Cardiovasc Electrophysiol 2000; 11: $736-740$.

15. Nielsen TD, Hamdan MH, Kowal RC, Barbera SJ, Page RL, Joglar JA. Effect of acute amiodarone loading on energy requirements for biphasic ventricular defibrillation. Am J Cardiol 2001; 88: 446-448.

16. Satomi K, Kurita T, Takatsuki S, Yokoyama Y, Chinushi M, Nitta T, et al. Does amiodarone therapy improve the prognosis of VT/VF patients after ICD implantation? NIPPON (The Nippon Amiodarone Therapy with ICD Viability Evaluation) pre-study (abstract). Circ J 2005; 69(Suppl): 136.

17. Pacifico A, Hohnloser SH, Williams JH, Tao B, Saksena S, Henry $\mathrm{PD}$, et al. Prevention of implantable-defibrillator shocks by treatment with sotalol: d,1-Sotalol Implantable Cardioverter-Defibrillator Study Group. N Engl J Med 1999; 340: 1855-1862.

18. Kuhlkamp V, Mewis C, Mermi J, Bosch RF, Seipel L. Suppression of sustained ventricular tachyarrhythmias: A comparison of d,l-sotalol with no antiarrhythmic drug treatment. J Am Coll Cardial 1999; 33: $46-52$.

19. Aiba T, Kurita T, Taguchi A, Shimizu W, Suyama K, Aihara N, et al. Long-term efficacy of empirical chronic amiodarone therapy in patients with sustained ventricular tachyarrhythmia and structural heart disease. Circ J 2002; 66: 367-371.

20. The CASCADE Investigators. Randomized antiarrhythmic drug therapy in survivors of cardiac arrest (the CASCADE Study). Am J Cardiol 1993; 72: 280-287.

21. McHorney CA, Kosinski M, Ware JE Jr. Comparisons of the costs and quality of norms for the SF-36 health survey collected by mail versus telephone interview: Results from a national survey. Med Care 1994; 32: 551-567.

22. Oyama N, Oyama N, Yoshiki H, Kamishima T, Nambu T, Tsutsui H, et al. Detection of amiodarone-induced pulmonary toxicity in supine and prone positions: High-resolution computed tomography study. Circ J 2005; 69: 466-470.

23. Bansch D, Castrucci M, Bocker D, Breithardt G, Block M. Ventricular tachycardias above the initially programmed tachycardia detection interval in patients with implantable cardioverter-defibrillators: Incidence, prediction and significance. J Am Coll Cardial 2000; 36: $557-565$.

24. Bansch D, Steffgen F, Gronefeld G, Wolpert C, Bocker D, Mletzko $\mathrm{RU}$, et al. The $1+1$ trial: A prospective trial of a dual- versus a singlechamber implantable defibrillator in patients with slow ventricular tachycardia. Circulation 2004; 110: 1022 - 1029.

25. The DAVID investigators. Dual-chamber pacing or ventricular backup pacing in patients with an implantable defibrillator: The dual chamber and VVI implantable defibrillator (DAVID) trial. JAMA 2002; 288: 3115-3123.

26. Freedman LS. Tables of the number of patients required in clinical trials using the logrank test. Stat Med 1982; 1: 121-129. 